\title{
Chronic Nicotine Self-Administration Augments Hypothalamic-Pituitary-Adrenal Responses to Mild Acute Stress
}

\author{
Hao Chen', Yitong Fu' and Burt M Sharp*,' \\ 'Department of Pharmacology, University of Tennessee Health Science Center, Memphis, TN, USA
}

\begin{abstract}
We investigated the effect of chronic nicotine self-administration (SA) on hypothalamic-pituitary-adrenal (HPA) hormonal responses to acute stressors. Adult male Sprague-Dawley rats were given access to nicotine $(0.03 \mathrm{mg} / \mathrm{kg})$ for $23 \mathrm{~h}$ per day for 20 days. On day I of acquisition of nicotine SA, plasma levels of both adrenocorticotropin and corticosterone were significantly increased I5-30 min after the first dose of nicotine. These hormonal changes were no longer significant on day 3 , when adrenocorticotropin levels were $<60 \mathrm{pg} / \mathrm{ml}$ and corticosterone levels were $<110 \mathrm{ng} / \mathrm{ml}$ during the hour after the first dose of nicotine. Chronic nicotine SA (20 days) significantly augmented (2-3-fold) both hormonal responses to mild foot shock stress ( $0.6 \mathrm{~mA}, 0.5 \mathrm{~s}$ per shock, 5 shocks per 5 min), but did not affect hormonal responses to moderate shock ( $1.2 \mathrm{~mA}, 0.5 \mathrm{~s}$ per shock, 5 shocks per $5 \mathrm{~min}$ ), lipopolysaccharide or immobilization. Similar data were obtained in Lewis rats. These results provide further support for the concept that chronic nicotine SA is a stressor. In alignment with the effects of other stressors, nicotine activated the HPA axis on the first day of SA, but desensitization occurred with repeated exposure. Furthermore, chronic nicotine SA selectively cross-sensitized the HPA response to a novel stressor. These observations suggest that nicotine may selectively increase the HPA response to stressors in human smokers.

Neuropsychopharmacology (2008) 33, 72 I-730; doi: I 0. I038/sj.npp. I 30I466; published online 6 June 2007
\end{abstract}

Keywords: nicotine self-administration; stress; ACTH; corticosterone; psychological stress; physical stress

\section{INTRODUCTION}

The complex interplay between cigarette smoking and stress has been intensively studied in the past decade. Psychosocial stressors, such as childhood abuse, low socioeconomic status, or other nonspecific stressors, have been consistently associated with increased risk for smoking initiation (Schepis and Rao, 2005). For example, a recent study (Finkelstein et al, 2006) showed that adolescents who experience higher baseline stress were more likely to become smokers. However, it is a matter of controversy whether smoking reduces or increases the response to stressors. Smokers usually report reduced stress levels after smoking (Coan, 1973; McArthur et al, 1958; Parrott, 1995); and some adolescents use smoking as a mechanism to cope with stressors (Koval et al, 2004). On the other hand, smokers, particularly during adolescence, reported significantly higher levels of perceived stress than nonsmokers (Croghan et al, 2006; Finkelstein et al, 2006), and quitting

*Correspondence: Dr BM Sharp, Department of Pharmacology, University of Tennessee Health Science Center, 874 Union Avenue, Memphis, TN 38163, USA, Tel: + I 901448 6000, Fax: + I 901 448 7206, E-mail: bsharp@utmem.edu

Received 16 February 2007; revised 5 April 2007; accepted 30 April 2007 smoking leads to reduced stress (Carey et al, 1993; Chassin et al, 2002; Cohen and Lichtenstein, 1990).

In animal studies, many laboratories, including ours, have reported that nicotine, the main psychoactive component of cigarettes, is a potent stimulus for the secretion of stressresponsive hormones, such as adrenocorticotropic hormone (ACTH) (Cam and Bassett, 1983b; Conte-Devolx et al, 1981; Matta et al, 1987), prolactin (Andersson et al, 1983; Sharp and Beyer, 1986), and corticosterone (CORT) (Balfour et al, 1975; Cam and Bassett, 1983a). We further demonstrated that nicotine indirectly induces ACTH secretion from the anterior pituitary by directly activating noradrenergic areas of the nucleus tractus solitarius (Matta et al, 1998; Zhao et al, 2007).

Complex bi-directional interactions between nicotine and stressors have been reported (Caggiula et al, 1998). Nicotine appears to affect stress responsiveness and stressors may modulate the intake of nicotine. These effects depend on the dose, duration, and route of nicotine administration, and on the modality of the stressor. The mode of nicotine delivery (eg forced $v s$ self-administered) is another determinant of its endocrine and neurotransmitter effects (Donny et al, 2000). All these interactions are further complicated by rapid desensitization to the stimulatory effects of nicotine on stress hormone secretion (Sharp and Beyer, 1986). 
Many previous animal studies of the hypothalamicpituitary-adrenal (HPA) axis utilized higher dosages of nicotine than those obtained by humans inhaling tobacco smoke (Faraday et al, 2003, 2005). High-intensity stressors, rarely experienced by smokers, also were commonly administered (Faraday et al, 2005; Stier et al, 2004). However, the effects of long-term nicotine self-administration (SA) on HPA responses to mild stressors, an experimental approach most germane to human smokers, has not been studied. We hypothesized that the long-term nicotine SA would enhance the HPA response to mild stressors. We evaluated this postulate in an established model of chronic nicotine SA with access to the drug for $23 \mathrm{~h} /$ day, using a dosage of nicotine (ie $\mathrm{mg} / \mathrm{kg}$ (body weight)/day) similar to the amount of daily nicotine intake by moderate smokers (Valentine et al, 1997). Since stress responsiveness is known to vary between rat strains, we evaluated Sprague-Dawley (SD) rats comprehensively and then made selective comparisons to Lewis rats. The inbred Lewis strain has atypical HPA responsiveness (Kosten and Ambrosio, 2002), and is vulnerable to the SA of nicotine and other abused substances (Brower et al, 2002).

In the current studies, self-administered nicotine initially stimulated the HPA axis in both strains; however, this was no longer evident by the third day of nicotine SA. We found that chronic (20 days) nicotine SA selectively amplified the HPA response to mild, but not moderate foot shock stress. Nicotine SA did not modify the HPA responses to immobilization stress or i.v. lipopolysaccharide (LPS).

\section{MATERIALS AND METHODS}

\section{Nicotine SA}

Adult male SD or Lewis rats (300-350 g, Harlan, Madison, WI) were given 7 days to recover from shipping and acclimated to a reversed 12:12 h light/dark cycle. Standard rat chow and water were provided ad libitum throughout the experiment. Nicotine solutions $(\mathrm{pH}=7.2$; calculated as free base) were freshly prepared for each cohort of animals in order to deliver $0.03 \mathrm{mg} / \mathrm{kg}$ (body weight)/i.v. dose. SA was performed according to our previously published protocol with some modifications (Brower et al, 2002; Valentine et al, 1997). Briefly, rats received jugular and femoral cannulae and were then housed individually in operant chambers (Coulbourn Instruments, Allentown, PA). Each operant chamber was placed inside a sound-attenuating environmental enclosure, where it resided for the duration of the experiment. Each operant chamber contained two horizontal levers positioned $6 \mathrm{~cm}$ above the floor. A green cue light located $1 \mathrm{~cm}$ above each lever was illuminated only when nicotine was available. Lever presses were recorded and syringe pumps were controlled by computers and interfaces located in an adjacent room, using L2T2 or Graphic State software (Coulbourn Instruments). Rats were given 3-5 days to recover from the surgery, during which time rats received daily antibiotic injections (Baytril, $7.6 \mathrm{mg} / \mathrm{kg}$ (body weight) in $0.1 \mathrm{ml}$, i.v.) and hourly computer-driven aliquots $(50 \mu \mathrm{l})$ of heparin-containing $(200 \mathrm{IU} / \mathrm{ml})$ saline.

Rats were given access to nicotine SA $23 \mathrm{~h} /$ day for 20 days. They learned to self-administer nicotine without prior training, priming or food deprivation. One lever in the operant chamber was randomly designated as the active lever. On SA days, pressing the active lever elicited a computer-driven i.v. injection of nicotine $(0.03 \mathrm{mg} / \mathrm{kg}+$ $200 \mathrm{U}$ heparin in $50 \mu \mathrm{l}$ ) or vehicle (200 U heparin in saline) delivered over $0.81 \mathrm{~s}$ through the jugular catheter. Pressing the alternate (inactive) lever had no programmed consequence. To avoid over-dosing, each injection was followed by a $7 \mathrm{~s}$ period during which the green cue light was extinguished and lever pressing was recorded, but nicotine was not injected.

The final hour of the lights-on cycle (0900-1000) was reserved for housekeeping tasks, such as refreshing the nicotine solution, performing animal husbandry, resetting the computer program, and downloading data. During this interval, the doors of the environmental enclosures were opened and green cue lights were turned off. Levers were not retracted, and lever press activity was not recorded nor rewarded during this period. The patency of each jugular line was checked every 2-3 days. Rats with closed lines were excluded from data analysis. All procedures conformed to NIH (National Institute of Health) guidelines and were approved by the Institutional Animal Care and Use Committee at The University of Tennessee Health Science Center.

\section{ACTH and CORT Responses to Self-Administered Nicotine}

A separate cohort of rats was used in this experimental protocol and in all those described in the subsequent sections on stress. Both SD $(n=14$ for nicotine SA and $n=10$ for vehicle SA) and Lewis rats $(n=5$ for nicotine and vehicle SA) were used. On the first and third day of acquisition of nicotine SA, a femoral vein blood sample $(0.2 \mathrm{ml})$ was withdrawn immediately after the first selfadministered dose of nicotine or vehicle. Four more samples $(0.2 \mathrm{ml})$ were obtained at $15,30,45$, and $60 \mathrm{~min}$, and an equivalent volume of saline containing $200 \mathrm{U}$ heparin was injected after each blood sampling. We were not able to obtain blood samples from two nicotine SA SD rats on day 1 .

\section{Electric Foot Shock Stress}

On the test day (nicotine SA day 20), rats were allowed to continue routine nicotine SA, and the first femoral venous blood sample was obtained $60 \mathrm{~min}$ after the first active lever press for nicotine. Then five random mild $(0.6 \mathrm{~mA}, 0.5 \mathrm{~s}$ per shock) or moderate ( $1.2 \mathrm{~mA}, 0.5 \mathrm{~s}$ per shock) electric shocks were delivered in 5 min through a floor grid (Model H13-16, Coulbourn Instruments). A total of 24 SD rats were used ( $n=6$ for each combination of shock intensity and SA treatment). We were not able to obtain blood samples from $1-2$ rats in some groups. In addition, 18 Lewis rats $(n=9$ for each SA treatment group) were tested using the mild foot shock procedure. Lever press behavior was virtually unaffected by foot shock stress, most likely due to the low intensity of the shock delivered. For example, the number of active lever presses were $4.6 \pm 0.7$ and $5.0 \pm 1.6$ for the hour before and after moderate foot shock, respectively. 


\section{Endotoxin Stress}

A single i.v. dose of LPS (L-4005; Sigma, St Louis, MI) 0.5 or $50.0 \mu \mathrm{g} / \mathrm{kg}$ (body weight) (in $0.3 \mathrm{ml}$ saline) was administered. On the test day (nicotine SA day 20), rats were allowed routine access to nicotine. Baseline femoral venous blood sampling, performed $60 \mathrm{~min}$ after the first active lever press, was followed by the injection of LPS delivered in $0.3 \mathrm{ml}$ saline through the femoral catheter. Control rats received the same volume of pyrogen-free saline. A total of $28 \mathrm{SD}$ rats were used ( $n=7$ for each combination of shock intensity and SA treatment). We were not able to obtain blood samples from 1-2 rats in some groups.

\section{Immobilization Stress}

The immobilization device was constructed from a $20 \mathrm{oz}$ Diet Coca Cola bottle by removing both the cap and half the cylinder comprising the lower $2 / 3$ of the bottle. On the test day (nicotine SA day 20), a baseline femoral venous blood sample was obtained $60 \mathrm{~min}$ after the first active lever press $(0 \mathrm{~min})$, and then a rat was wrapped in canvas (head not included) and placed in the prone position within the cylindrical part of the bottle for $30 \mathrm{~min}$. Subsequent samples were withdrawn at $30,60,120$, and $180 \mathrm{~min}$ after the initiation of immobilization. A total of $14 \mathrm{SD}$ rats were used ( $n=7$ for each SA treatment). We were not able to obtain blood samples from one nicotine SA rat.

\section{ACTH and CORT Radioimmunoassays}

Blood was collected into ice-cold tubes containing ethylenediaminetetraacetic acid (EDTA) $(150 \mathrm{mg} / \mathrm{ml})$, spun at 2800 r.p.m. at $4^{\circ} \mathrm{C}$, and stored at $-80^{\circ} \mathrm{C}$. ACTH and CORT were measured on unextracted plasma samples, according to previously published methods (Sharp and Beyer, 1986), using the ImmuChem ${ }^{\mathrm{TM}}$ double antibody ${ }^{125} \mathrm{I}$-corticosterone RIA kit and the RSL ${ }^{125}$ I-hACTH kit (MP Biomedicals, NY).

\section{Statistics}

Data were presented as mean \pm SEM, and were analyzed using repeated-measures analysis of variance (ANOVA), where time or day was treated as a within-subject factor. Statistical significance was assigned for $p<0.05$.

\section{RESULTS}

\section{Nicotine SA}

As reported previously (Brower et al, 2002; Parker et al, 2004; Valentine et al, 1997), SD and Lewis rats acquired nicotine $(0.03 \mathrm{mg} / \mathrm{kg}) \mathrm{SA}$ without prior training, priming or food deprivation when the drug was available $23 \mathrm{~h}$ /day. Similar levels of nicotine SA were achieved by the rats used throughout these experiments. The number of lever presses recorded for one representative group of SD rats was shown in Figure 1. Repeated-measures ANOVA found a significant main effect of treatment (nicotine $v s$ vehicle) $\left(\mathrm{F}_{1,9}=7.28\right.$, $p<0.05$ ) on the number of lever presses. The interaction between day and lever was also significant $\left(\mathrm{F}_{19,171}=2.44\right.$, $p<0.01)$. There was a significant difference between the

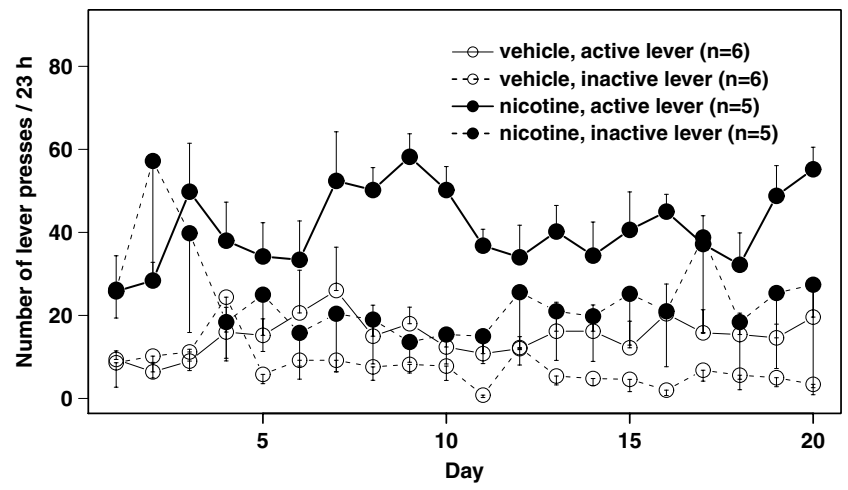

Figure I Nicotine SA in SD rats. Adult male SD rats were housed in operant chambers and given access to two levers for 20 days. During each consecutive session ( $23 \mathrm{~h} /$ day), pressing the active lever was followed by a rapid i.v. infusion of nicotine $(0.03 \mathrm{mg} / \mathrm{kg}$ (body weight)) and then a $7 \mathrm{~s}$ interval during which nicotine was not delivered if the active lever was pressed. Pressing the inactive lever had no programmed consequence. Nicotine SA was acquired without prior training, priming or food deprivation. ANOVA found a significant main effect of treatment (nicotine vs vehicle: $F_{1,9}=7.28, p<0.05$ ). The number of active lever presses were significantly higher than that of the inactive lever presses in the nicotine SA group (main effect of lever: $F_{1,4}=13.58, p<0.01$ ) but not in the vehicle SA group (main effect of lever: $F_{1,5}=1.52, p>0.05$ ). The number of active lever presses per day in the nicotine SA group was $2.93 \pm 0.21$ fold higher than that of the vehicle SA group (main effect of treatment: $F_{1,9}=10.50$, $p<0.01$ ). The data were from a representative group of rats.

number of active and inactive lever presses $\left(F_{1,4}=13.58\right.$, $p<0.01)$ in the nicotine, but not in the vehicle SA group $\left(F_{1,5}=1.52, p>0.05\right)$. The number of active lever presses per day in the nicotine SA group was $2.93 \pm 0.21$-fold higher than that of the vehicle $S A$ group $\left(F_{1,9}=10.50, p<0.01\right)$.

\section{ACTH and CORT Responses during the Acquisition of Nicotine SA in SD Rats}

We measured plasma ACTH and CORT levels in SD rats immediately after the first dose of nicotine was delivered on the first and third days of access to nicotine SA (Figure 2). On day 1, repeated-measures ANOVA showed that ACTH levels were significantly greater in the nicotine SA group $\left(F_{1,20}=10.50, p<0.01\right)$, but the effect of time was not significant $\left(\mathrm{F}_{4,80}=0.865, p>0.05\right)$. The treatment $\times$ time interaction was not significant either $\left(\mathrm{F}_{4,80}=0.931\right.$, $p>0.05)$. Similarly, nicotine SA had a significant effect on CORT levels $\left(\mathrm{F}_{1,20}=19.70, p<0.001\right)$, although CORT was unaffected by time $\left(\mathrm{F}_{4,80}=1.15, p>0.05\right)$, and the treatment $\times$ time interaction was insignificant $\left(\mathrm{F}_{4,80}=2.98\right.$, $p>0.05)$. On the third day of SA, neither treatment nor time affected ACTH levels $\left(\mathrm{F}_{1,22}=0.28, \mathrm{~F}_{4,88}=0.37\right.$, respectively, $p>0.05$ for both). Similarly, neither treatment nor time affected CORT levels on day 3 of $\mathrm{SA}\left(\mathrm{F}_{1,22}=1.65\right.$, $\mathrm{F}_{4,88}=2.03$, respectively, $p>0.05$ for both).

\section{Nicotine SA Augmented HPA Hormonal Responses to Mild but not Moderate Intensity Electric Foot Shock in SD Rats}

We tested the effect of chronic nicotine SA on the hormonal response to randomized mild $(0.6 \mathrm{~mA}, 5$ times in $5 \mathrm{~min})$ or moderate (1.2 mA, 5 times in $5 \mathrm{~min}$ ) electric foot shock. In 

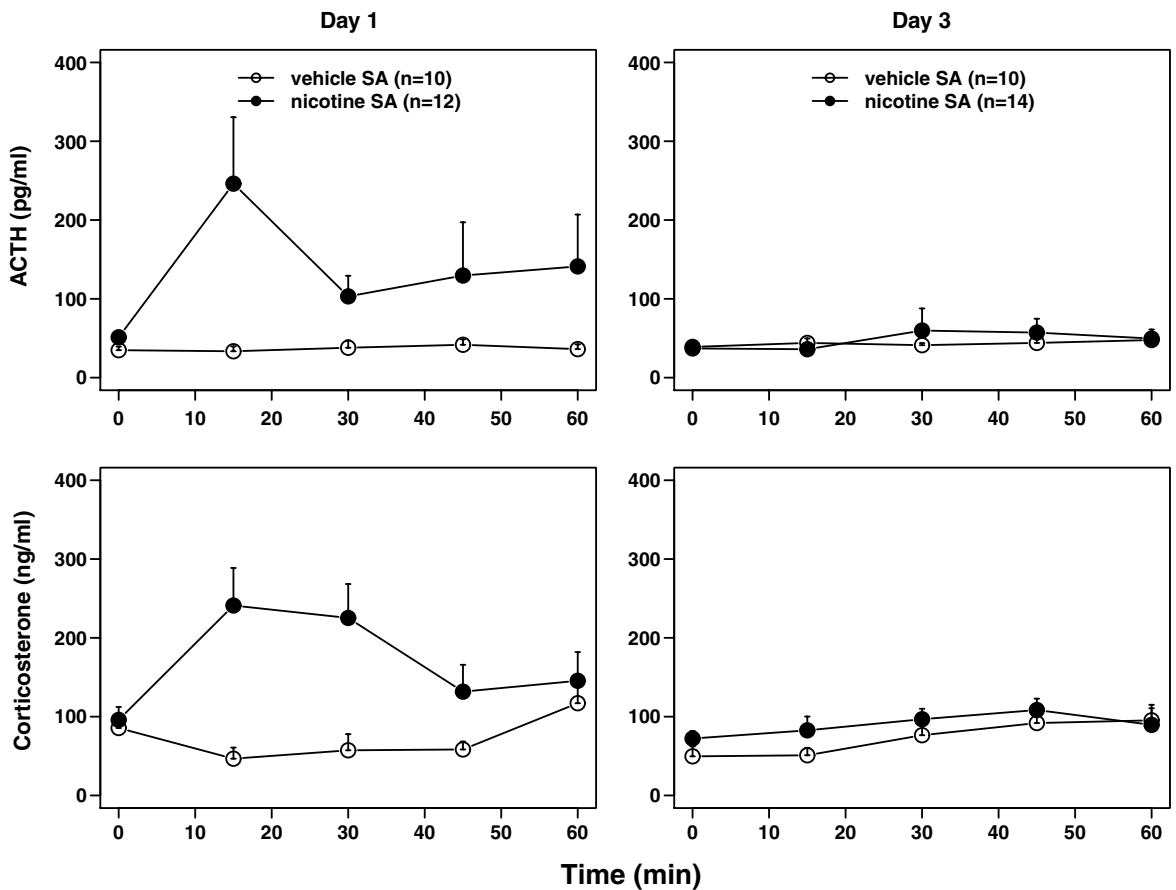

Figure 2 Plasma adrenocorticotropic hormone (ACTH) and corticosterone (CORT) responses on the first and third day of nicotine self-administration $(\mathrm{SA})$ in SD rats. Blood samples were collected through a femoral cannula on the first and third days of nicotine SA at the time of the first active lever press (0 min), and I5, 30, 45, and 60 min thereafter. On the first day, nicotine SA induced significant increases in plasma ACTH (left upper panel) and CORT (left lower panel) levels compared to rats self-administering vehicle (repeated-measures ANOVAs showed main effects of treatment: $A C T H, F_{1,20}=10.50$, $p<0.0 \mathrm{I}$; $\mathrm{CORT}, \mathrm{F}_{1,20}=19.70, p<0.00 \mathrm{I}$ ). On the third day of nicotine SA (right panels), no significant main effects were detected. Hormone concentrations were expressed as mean $\pm \mathrm{SEM}$.

rats self-administering nicotine on day 20 , mild shocks were delivered $60 \mathrm{~min}$ after the first nicotine injection of the day. The delivery of the electric shocks in the vehicle SA group was paired with the nicotine SA rats. Figure 3 shows that mild foot shock significantly elevated plasma CORT levels $\left(\mathrm{F}_{4,36}=3.27, p<0.05\right.$ for main effect of time, repeatedmeasures ANOVA). There was no time $\times$ treatment interaction $\left(\mathrm{F}_{4,36}=1.00, p>0.05\right)$; therefore, CORT increased over time in both treatment groups. Most importantly, nicotine SA significantly augmented the CORT and ACTH responses to mild foot shock $\left(\mathrm{F}_{1,9}=14.35, p<0.001\right.$ and $\mathrm{F}_{1,9}=10.15, p<0.001$, respectively).

In separate groups of rats, moderate electric foot shocks were also delivered using the same schedule of delivery as in the preceding experiment. In vehicle SA rats, moderate shock induced greater plasma ACTH and CORT levels than mild foot shock $\left(\mathrm{F}_{1,9}=24.04\right.$ and 19.69, for ACTH and CORT, respectively, and $p<0.001$ for both) (Figure 3). Moderate foot shock had the same effect on ACTH in the nicotine and vehicle SA groups $\left(\mathrm{F}_{1,7}=0.07, p>0.05\right)$. There was a significant main effect of time on ACTH levels $\left(\mathrm{F}_{4,28}=6.62, p<0.001\right)$, and the time $\times$ treatment interaction was not significant $\left(\mathrm{F}_{4,28}=0.44, p>0.05\right)$. Similar results were obtained for plasma CORT responses. The main effect of time was significant $\left(\mathrm{F}_{4,28}=14.6, p<0.001\right)$, while the main effect of treatment $\left(\mathrm{F}_{1,7}=0.68, p>0.05\right)$ and the time $\times$ treatment interaction $\left(\mathrm{F}_{4,28}=1.37, p>0.05\right)$ were not significant.

Taken together, these data showed that ACTH and CORT responses to foot shock depend on the intensity of the electric current and on prior exposure to nicotine SA. A greater elevation of both ACTH and CORT was stimulated by moderate than mild foot shock. Nicotine SA selectively augmented the ACTH and CORT responses to mild but not to moderate foot shock in SD rats.

\section{Nicotine SA did not Affect the Hormonal Response to Different Doses of LPS in SD Rats}

We evaluated the effect of chronic nicotine SA on HPA responses induced by a high dose of LPS $(50.0 \mu \mathrm{g} / \mathrm{kg}$, i.v. $)$. On day 20, LPS was injected $60 \mathrm{~min}$ after the first selfadministered injection of nicotine. The delivery of LPS in the vehicle SA group was paired with the nicotine SA rats. LPS increased plasma ACTH levels (Figure 4, left panels). Repeated-measures ANOVA found that the main effect of time was significant $\left(\mathrm{F}_{4,44}=24.41, p<0.001\right)$. However, the effect of SA treatment $\left(\mathrm{F}_{4,44}=2.57, p>0.05\right)$ and the time by treatment interaction $\left(\mathrm{F}_{4,44}=2.21, p>0.05\right)$ were not significant. The CORT responses were similar to ACTH. The effect of time was significant $\left(\mathrm{F}_{4,44}=21.11, p<0.001\right)$, whereas the effect of SA treatment $\left(\mathrm{F}_{1,11}=0.69, p>0.05\right)$ and the time $\times$ treatment interaction $\left(F_{4,44}=0.62, p>0.05\right)$ were not significant. The effect of another LPS dose $(12.5 \mu \mathrm{g} / \mathrm{kg})$ was also tested and the results were similar (data not shown). Therefore, chronic nicotine SA did not modify the HPA responses to either high $(50 \mu \mathrm{g} / \mathrm{kg})$ or moderate $(12.5 \mu \mathrm{g} / \mathrm{kg})$ doses of LPS.

Since nicotine SA amplified HPA responses to mild but not moderate foot shock stress, we also evaluated whether nicotine SA would affect HPA responses to a very low dose of LPS $(0.5 \mu \mathrm{g} / \mathrm{kg})$, which itself induced the minimal 

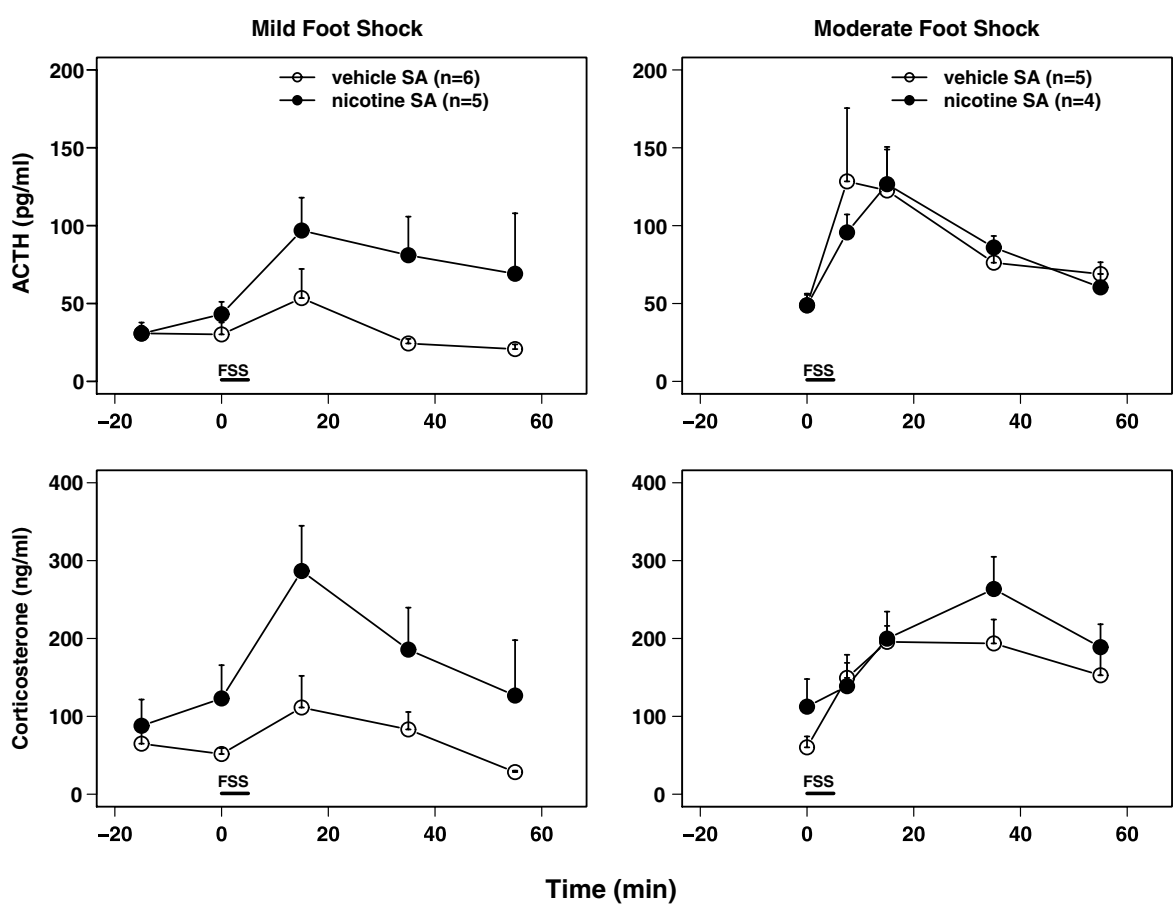

Figure 3 Long-term nicotine SA augmented adrenocorticotropic hormone (ACTH) and corticosterone (CORT) responses to mild but not to moderate foot shock in SD rats. On the 20th day of nicotine SA, baseline blood samples were collected 45 and 60 min after the first active lever press of the day (shown as -15 and $0 \mathrm{~min}$ ) and then foot shock stress (FSS) was administered. The delivery of FSS in the vehicle SA group was paired with the nicotine SA rats. Nicotine SA significantly enhanced both the ACTH and CORT responses to mild FSS (0.6 mA, randomly delivered 5 times per 5 min; left panels) (repeated-measures ANOVAs showed main effects of treatment: ACTH, $F_{1,9}=10.15, p<0.001$; CORT, $F_{1,9}=\mid 4.35$, $p<0.00$ I). In a separate group, nicotine SA did not modulate the ACTH and CORT responses to moderate FSS (I.2 mA, randomly delivered 5 times per 5 min; right panels). Hormone concentrations were expressed as mean \pm SEM.

hormonal responses. Indeed, in the vehicle control groups (Figure 4, right panels), the CORT response to $0.5 \mu \mathrm{g} / \mathrm{kg}$ LPS was significantly less than to $50 \mu \mathrm{g} / \mathrm{kg}$ LPS $\left(\mathrm{F}_{1,9}=15.69\right.$, $p<0.01)$. Very low dose LPS increased CORT levels minimally $\left(\mathrm{F}_{4,40}=8.92, p<0.001\right)$, although there was no effect of treatment $\left(\mathrm{F}_{1,10}=1.66, p>0.05\right)$ nor interaction between treatment and time $\left(\mathrm{F}_{4,40}=0.58, p>0.05\right)$. ACTH levels were not affected by very low dose LPS (main effect of time: $\mathrm{F}_{4,40}=2.04, p>0.05$ ), nor was the effect of SA treatment significant $\left(\mathrm{F}_{1,10}=1.77, p>0.05\right)$. Therefore, very low dose LPS stimulated small but significant CORT responses. In contrast to mild foot shock stress, chronic nicotine SA did not modulate the HPA responses to this low dose LPS regimen. Taken together, these data demonstrate that chronic nicotine SA does not modulate HPA responsiveness to very low or high dosages of LPS.

\section{Nicotine SA did not Affect the Hormonal Response to Immobilization Stress in SD Rats}

We tested the effect of chronic nicotine SA on HPA hormonal responses to immobilization stress. On day 20 of SA, 60 min after the first self-administered dose of nicotine, rats were immobilized for $30 \mathrm{~min}$ in the prone position within their individual operant chambers. The schedule of blood withdrawal in the vehicle SA group was paired with the nicotine SA rats. Immobilization increased the ACTH levels in both SA treatment groups (Figure 5). Repeated-measures ANOVA showed a main effect of time $\left(\mathrm{F}_{4,44}=18.44, p<0.001\right)$. The effect of treatment
$\left(\mathrm{F}_{1,11}=0.07, p>0.05\right)$ and time $\times$ treatment interaction $\left(\mathrm{F}_{4,44}=0.29, p>0.05\right)$ were not significant. The CORT responses to immobilization were similar to ACTH. There was a main effect of time $\left(\mathrm{F}_{4,44}=41.77, p<0.001\right)$, but the effects of treatment $\left(\mathrm{F}_{1,11}=0.10, p>0.05\right)$ and time $\times$ treatment interaction were not significant $\left(\mathrm{F}_{4,44}=1.78, p>0.05\right)$. Therefore, chronic nicotine SA did not modulate the hormonal responses to immobilization stress.

\section{Effects of Nicotine SA on HPA Responses in Lewis Rats}

HPA responsiveness is known to vary among rat strains. Therefore, based on the foregoing data obtained in SD rats, selective comparisons were made to inbred Lewis rats, known for their atypical HPA responsiveness (Kosten and Ambrosio, 2002) and sensitivity to nicotine SA (Brower et al, 2002). HPA responses to nicotine SA on the first and third day of access to nicotine and to mild foot shock were evaluated. Since ACTH and CORT demonstrated corresponding changes to either chronic nicotine SA or stressors in SD rats, and chronic nicotine SA augmented both the ACTH and CORT responses, we only measured CORT levels in Lewis rats.

On the first day of access to nicotine SA (Figure 6a), the first self-administered dose of nicotine significantly increased the CORT levels $\left(\mathrm{F}_{1,8}=9.96, p<0.01\right)$ over time $\left(\mathrm{F}_{4,32}=4.96, p<0.01\right)$ in comparison to the vehicle control group. On the third day of nicotine SA (Figure 6b), the effect of time on CORT levels was not significant $\left(F_{4,32}=2.12, p>0.05\right)$. These results are in agreement with 

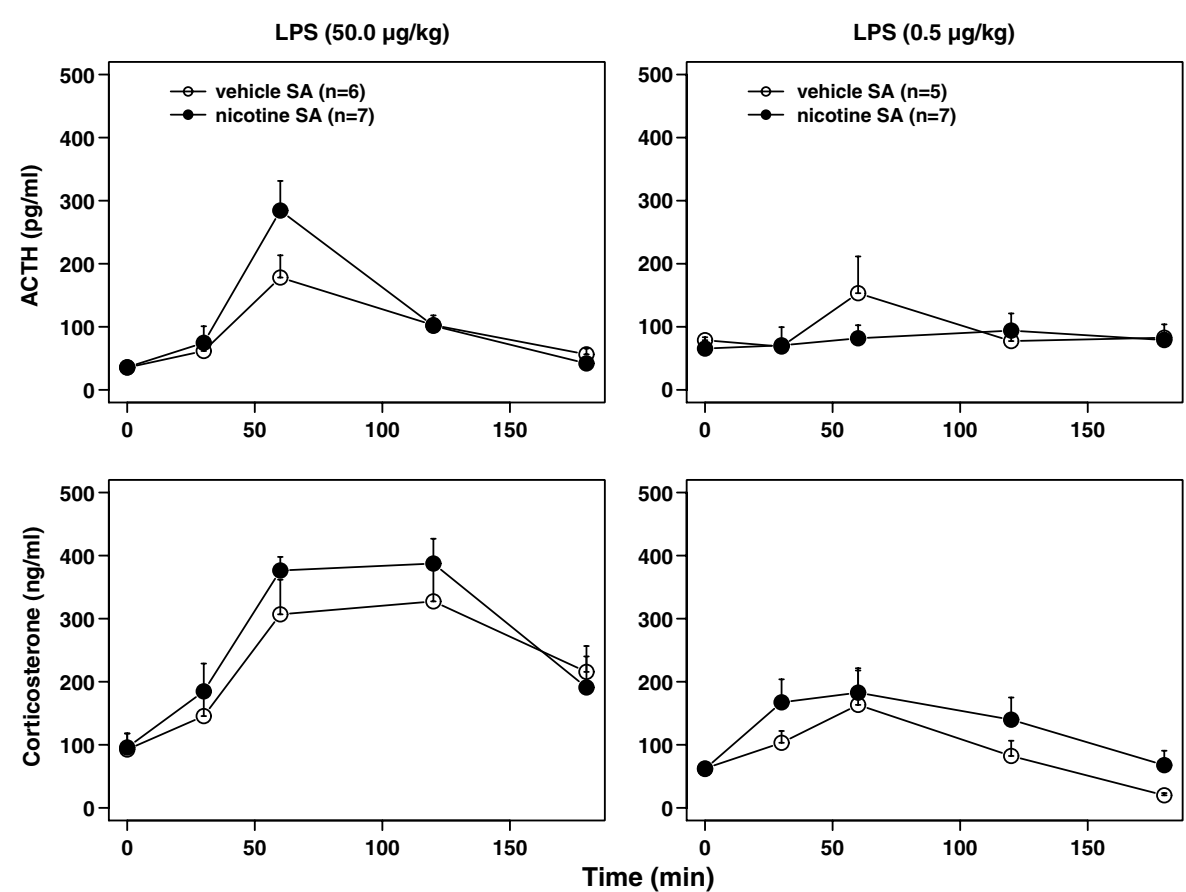

Figure 4 Long-term nicotine SA did not modulate adrenocorticotropic hormone (ACTH) and corticosterone (CORT) responses to lipopolysaccharide (LPS) in SD rats. On the 20th day of nicotine SA, a baseline blood sample was collected 60 min after the first active lever press of the day (shown as 0 min) Thereafter, LPS ( 50.0 or $0.5 \mu \mathrm{g} / \mathrm{kg}$, i.v.) was injected, and blood samples were collected at the time intervals specified in the figures. LPS $50.0 \mu \mathrm{g} / \mathrm{kg}$ significantly increased ACTH (left upper panel) and CORT (left lower panel) levels (repeated-measures ANOVAs showed main effects of time: ACTH, $\mathrm{F}_{4,44}=24.4 \mathrm{I}$, $p<0.00$ I; CORT, $F_{4,44}=21.1 \mathrm{I}, p<0.00 \mathrm{I}$ ). However, nicotine SA did not affect the hormonal responses to LPS $50.0 \mu \mathrm{g} / \mathrm{kg}$ (main effects of treatment: $\mathrm{ACTH}, \mathrm{F}_{4,44}=2.57, p>0.05$; CORT, $\mathrm{F}_{4,44}=0.69, p>0.05$ ). LPS $0.5 \mu \mathrm{g} / \mathrm{kg}$ did not affect ACTH (right upper panel) levels (main effect of time: $F_{4,40}=2.04$, $p>0.05$ ), although CORT (right lower panel) levels increased (main effect of time: $F_{4,40}=8.92, p<0.00 \mathrm{l}$ ). There were no effects of nicotine SA on hormonal responses to LPS $0.5 \mu \mathrm{g} / \mathrm{kg}$ (main effects of treatment: $\mathrm{ACTH}, \mathrm{F}_{1,10}=1.77, p>0.05 ; \mathrm{CORT}, \mathrm{F}_{1,10}=1.66, p>0.05$ ). Hormone concentrations were expressed as mean \pm SEM.
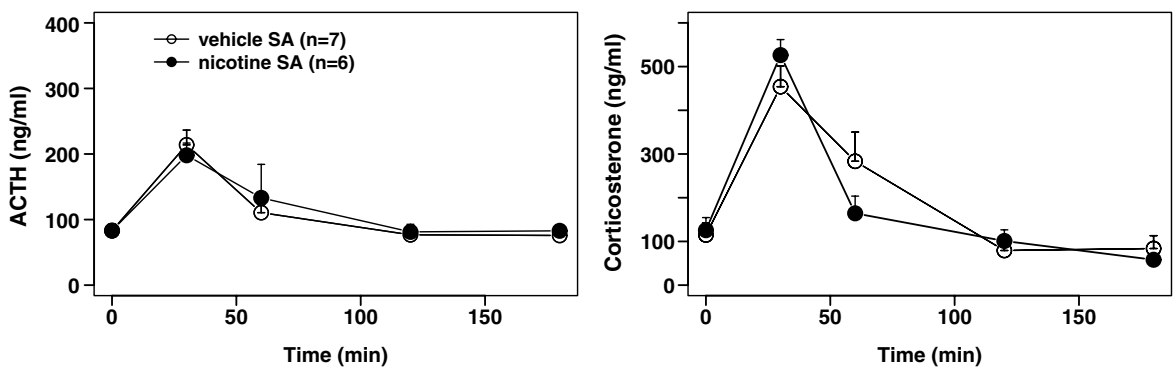

Figure 5 Long-term nicotine SA did not modulate adrenocorticotropic hormone (ACTH) and corticosterone (CORT) responses to immobilization in SD rats. On the 20th day of self-administration (SA), baseline blood sample was collected 60 min after the first active lever press of the day (shown as 0 min) Rats were then immobilized for $30 \mathrm{~min}$, and blood was sampled at the time intervals shown. Immobilization increased ACTH and CORT levels in both SA treatment groups (main effects of time: $A C T H, F_{4,44}=18.44, p<0.00 \mathrm{I}$; CORT, $F_{4,44}=41.77, p<0.00 \mathrm{I}$ ). However, nicotine $S A$ did not affect hormonal responses to immobilization (main effects of treatment: $A C T H, F_{1,11}=0.07, p>0.05 ; C O R T, F_{1,11}=0.10, p>0.05$ ). Hormone concentrations were expressed as mean $\pm \mathrm{SEM}$.

those obtained in SD rats. Similar to the absence of a CORT response in SD rats on day 3, repeated-measures ANOVA found that CORT was not significantly increased by the first dose of nicotine (Figure $6 \mathrm{~b} ; \mathrm{F}_{1,8}=3.74, p>0.05$ ) on day 3 .

On day 20 of nicotine SA (Figure 6c), mild foot shock significantly elevated CORT levels in Lewis rats $\left(\mathrm{F}_{5,88}=4.37\right.$, $p<0.01$ for the main effect of time). Nicotine SA significantly amplified this CORT response $\left(\mathrm{F}_{1,16}=8.50\right.$, $p<0.01)$. No treatment $\times$ time interaction was detected $\left(\mathrm{F}_{5,88}=1.29, p>0.05\right)$; therefore, mild foot shock increased CORT in both groups, and the response was greater in animals self-administering nicotine. Two differences were observed in the CORT responses in Lewis vs SD rats. First, the peak response occurred $15 \mathrm{~min}$ after shock in SD rats, and not until $30 \mathrm{~min}$ in the Lewis strain. Second, 15-60 min post foot shock, nicotine amplified the CORT response by $290 \%$ in SD rats compared to $138 \%$ in Lewis rats.

\section{DISCUSSION}

These studies in SD and Lewis male rats demonstrate that nicotine consistently stimulated the release of HPA hormones (ie ACTH and CORT) on the first but not the 
a

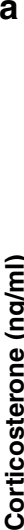

b Response to nicotine on day 3

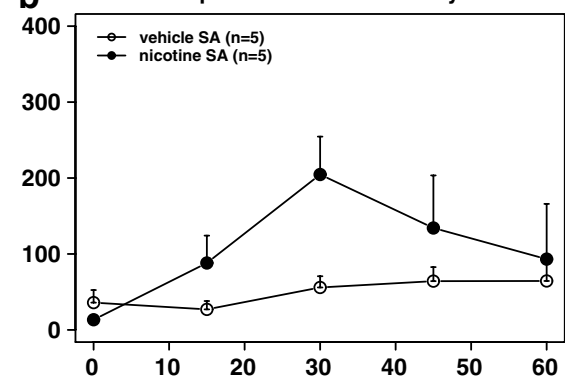

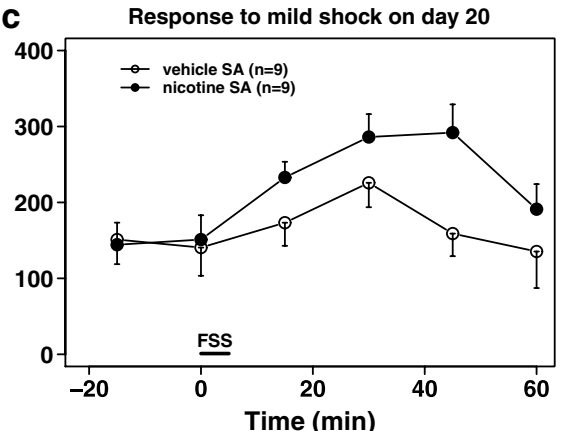

Figure 6 Corticosterone (CORT) responses to nicotine self-administration (SA) and to mild foot shock stress in Lewis rats. In the experiments shown in (a) and (b), blood samples were collected through a femoral cannula on the first and third days of nicotine SA at the time of the first active lever press (0 min), and 15, 30, 45, and 60 min thereafter. On the first day, nicotine SA induced significant increases in CORT levels (a) compared to rats selfadministering vehicle (repeated-measures ANOVA showed main effects: treatment, $F_{1,8}=9.96, p<0.01$; time, $F_{4,32}=4.96$, $p<0.01$ ). On the third day of nicotine SA (b), CORT was not significantly increased by the first dose of nicotine $\left(F_{1,8}=3.74, p>0.05\right)$. (c) shows CORT responses to mild foot shock stress (FSS). On the 20th day of nicotine SA, baseline blood samples were collected 45 and 60 min after the first active lever press of the day (shown as - I5 and $0 \mathrm{~min}$ ) and then FSS was administered. The delivery of FSS in the vehicle SA group was paired with the nicotine SA rats. Mild FSS (0.6 mA, randomly delivered 5 times per $5 \mathrm{~min}$ ) significantly increased CORT levels (main effect of time: $F_{5,88}=4.37, p<0.0$ I). Nicotine SA significantly amplified the CORT response to FSS (main effect of treatment: $F_{1,16}=8.50, p<0.01$ ). Hormone concentrations were expressed as mean $\pm S E M$.

third day of SA. We also found that long-term (20 days) nicotine SA selectively enhanced the hormonal response to mild but not moderate foot shock stress. In contrast, chronic nicotine SA did not affect the hormonal response to endotoxin (LPS) or immobilization stress.

Nicotine is a potent stimulus for the stress-responsive HPA axis that elicits the release of the stress hormones, ACTH (Cam and Bassett, 1983b; Matta et al, 1987) and CORT (Balfour et al, 1975; Cam and Bassett, 1983a). We have previously identified a polysynaptic pathway through which nicotine acutely stimulates the HPA axis (Matta et al, 1998). Corticotropin-releasing factor (CRF) neurons in the hypothalamic paraventricular nucleus (PVN), which are pivotal regulators of ACTH secretion, are not directly activated by nicotine (Matta et al, 1987). Instead, nicotine indirectly activates PVN CRF neurons (Matta et al, 1990) by stimulating a cascade of neurotransmitters in the nucleus tractus solitarius (NTS) that activate noradrenergic projections to the PVN (Zhao et al, 2007).

There is a substantial body of evidence supporting the concept that nicotine SA is a stressor. Acute nicotine activates a wide range of brain areas involved in regulation of the stress response (eg PVN, NTS, cingulate cortex, amygdala). Our finding that self-administered nicotine induced significant activation of the HPA axis, a cardinal dimension of the stress response, on the first day of SA is in agreement with previous reports that utilized a limited access model of nicotine SA (Donny et al, 2000). Evidence that long-term nicotine SA chronically stimulated the release of the stress-responsive neurotransmitter, norepinephrine (NE), in the PVN and amygdala (Fu et al, 2003), provides additional support for the concept that nicotine SA is a chronic stressor.

When the same stressor is administered repeatedly, activation of the HPA axis by subsequent exposure to this stressor (ie homotypic stressor) often diminishes. As little as one exposure may be sufficient to induce this desensitization, as shown for restraint, immobilization, foot shock and LPS (Armario et al, 2004). Early studies found that a single forced nicotine $(0.5 \mathrm{mg} / \mathrm{kg}$, i.p.) injection desensitized the ACTH response to a second injection of nicotine $(1.0 \mathrm{mg} / \mathrm{kg}$, i.p.) administered $1 \mathrm{~h}$ later (Sharp and Beyer, 1986). The current results, showing desensitization of the HPA response to self-administered nicotine by the third day of SA, link nicotine SA with the desensitization to other chronic stressors, further supporting the idea that selfadministered nicotine is a chronic stressor.

There are several potential mechanisms that might account for this desensitization. One potential mechanism would reflect pharmacokinetic changes. Previous studies have shown that nicotine metabolism is unaffected by chronic exposure to nicotine in animals (Baer et al, 1980; Marks et al, 1983) or humans (Benowitz and Jacob, 1993). However, recent studies found that CYP2B6, an enzyme involved in nicotine metabolism, is selectively induced in the brain but not in the liver (Lee et al, 2006). Therefore, an 
increased rate of brain nicotine metabolism could conceivably be a factor in the desensitization of the HPA axis response to chronic nicotine. Another potential mechanism involves NE. Since NE is essential for the release of ACTH and CORT by nicotine (Matta et al, 1998), desensitization might reflect a reduction in PVN NE release. In two models of nicotine delivery, forced or self-administered, we have previously measured the PVN NE response to multiple doses of nicotine. There was only partial desensitization ( $\sim 50 \%$ ) of the PVN NE response to a second injection of experimenter-administered nicotine, delivered $100 \mathrm{~min}$ after the first dose (Matta et al, 1995). However, the NE response to subsequent nicotine injections did not desensitize further. In nicotine self-administering animals, the first dose of nicotine increased PVN NE release to a similar extent on both day 1 and day 9 of the nicotine SA (Fu et al, 2001). Therefore, complete desensitization of the ACTH and CORT responses, as observed in SD rats on day 3 of nicotine $\mathrm{SA}$, is not due to deficient PVN NE secretion.

In the $23 \mathrm{~h}$ SA model used herein, the venous nicotine concentration was in the range of $20-40 \mathrm{ng} / \mathrm{ml}$ (LeSage et al, 2002), a level similar to that detected in chronic human smokers (10-40 ng/ml) (Benowitz and Jacob, 1984). At these concentrations, nicotine is known to activate and desensitize nicotinic acetylcholinergic receptors (nAChRs). Although we cannot rule out the possibility that such changes in $\mathrm{nAChR}$ function are involved in desensitization of the HPA response to self-administered nicotine, this is not likely to occur in the NTS, because the PVN NE response to the first self-administered dose of nicotine is similar on day 1 and day 9 of chronic nicotine SA (above). Alternatively, the posterior paraventricular thalamus (pPVTh) might be involved. pPVTh is required for the desensitization of HPA responses to repeated restraint stress (Bhatnagar et al, 2002). Since the pPVTh expresses nAChR subunits (eg $\alpha 4, \alpha 7$, and $\beta 2$ ) (Ray et al, 2005), it is conceivable that desensitization of PVTh nAChRs might be involved in the desensitization of HPA responsiveness to nicotine SA.

Regardless of the mechanism of desensitization, the current results from two rat strains suggest that nicotine activates the HPA axis when initially administered, and this effect desensitizes by day 3. This is in agreement with data showing that the HPA axis of chronic smokers was not stimulated (Gilbert et al, 1992; Mendelson et al, 2005) by cigarettes containing the usual amount of nicotine (eg approximately $1-1.5 \mathrm{mg}$ per cigarette) (Matta et al, 2007). Taken together, the present report and human studies indicate that daily intake of nicotine by established tobacco smokers is unlikely to activate the HPA axis.

Human smokers routinely experience diverse stressors, virtually on a daily basis. Animal studies have shown that myriad stressors activate the PVN, eventuating in the secretion of ACTH and CORT, through distinct neural mechanisms and pathways that subserve the various modalities of stress; this is especially applicable to physical (eg LPS) vs psychological (eg foot shock, restraint) stressors. For example, an acute peripheral injection of LPS stimulated the PVN, depending on prostaglandins and NE (Chuluyan et al, 1992; Ericsson et al, 1994). In contrast, activation of the HPA axis by psychological stressors, such as foot shock and restraint, did not require hypothalamic prostaglandins (Wan et al, 1994) nor NE from the brainstem (Chuluyan et al, 1992; Li et al, 1996). Instead, the primary brain regions mediating the HPA response to psychological stressors appear to be the cortex (eg prefrontal) and limbic system (eg amygdala) (Dayas et al, 2004; Sawchenko et al, 2000).

In addition to the mode of stress, its intensity is an important determinant of the central nervous system (CNS) response. For example, while high-dose LPS $(100 \mu \mathrm{g} / \mathrm{kg})$ or high-intensity foot shocks activated c-fos expression in locus coeruleus, a lower dose of LPS $(20 \mu \mathrm{g} / \mathrm{kg})$ or lower intensity foot shock did not (Goodwin et al, 1997; Stone et al, 2006; Wan et al, 1994). Since the stressors routinely experienced by smokers are relatively mild, we tested the effect of chronic nicotine SA on the HPA stress response to three different stressors, using different intensities of stressor, where possible. Two low-intensity electric foot shock paradigms and two LPS doses were evaluated. The intensity of immobilization stress, which involves handling and uniform physical contact between the apparatus and the rat, could not be modulated. The low-intensity application of LPS or foot shock induced minimal HPA activation in the control group (eg vehicle SA). Chronic nicotine SA selectively enhanced the hormonal responses to mild foot shock, whereas LPS, moderate-intensity foot shock (Figure 3) and immobilization stress (Figure 5) were unaffected by nicotine SA. In the respective control groups, moderate-intensity foot shock and immobilization both elevated ACTH and CORT to a significantly greater extent than did mild foot shock. Thus, the efficacy of nicotine SA in augmenting the HPA response appears to be specific to the stressor (eg foot shock) and limited by the intensity of that stressor. At higher stimulus intensities, a particular stressor may exceed the modulatory capacity of selfadministered nicotine.

Previous studies demonstrated that a chronic stressor can enhance the HPA response to a novel stressor (Bhatnagar and Dallman, 1998; Ma et al, 1999). Here, too, the pPVTh, which is essential for desensitization to a homotypic stressor (see the above), was required for the crosssensitization to a heterotypic stressor (eg restraint after repeated cold) (Bhatnagar and Dallman, 1998). The augmentation of HPA responsiveness to mild foot shock by chronic nicotine SA is congruent with cross-sensitization to a heterotypic stressor. Hence, the neural circuitry underlying cross-sensitization, including the pPVTh, might participate in the amplification of HPA responsiveness by nicotine SA.

Although the specificity for foot shock is an unexpected dimension of the cross-sensitization induced by nicotine $\mathrm{SA}$, it is consistent with an emerging viewpoint on the HPA axis that emphasizes its discriminative rather than ubiquitous responsiveness to diverse stressors (Deak et al, 2005; Li et al, 1996). Such specificity might depend on neuronal plasticity induced by nicotine within brain areas that are preferentially involved in mediating the stress response to psychological stressors. Medial prefrontal cortex (mPFC) is a brain region that might be modified by chronic nicotine SA. $\mathrm{mPFC}$ is an inhibitory regulator of the ACTH/CORT response to a psychological stressor (Radley et al, 2006). Indeed, foot shock induced c-fos expression in mPFC (ie cingulate cortex) (Passerin et al, 2000; Wan et al, 1994), 
whereas LPS affected the PVN and NTS, and not mPFC (Stone et al, 2006; Takemura et al, 1997). Nicotine SA has been shown to activate mPFC (Pagliusi et al, 1996). Since mPFC provides inhibitory modulation of the ACTH/CORT response (Radley et al, 2006), neuronal adaptation to longterm nicotine SA might attenuate this inhibition, thereby enhancing the HPA response to a heterotypic stressor (ie mild foot shock after chronic nicotine SA).

We tested two rat strains in these experiments. Despite the atypical stress response reported in the Lewis strain (Kosten and Ambrosio, 2002), similar responses were found in both Lewis and SD strains. The ACTH/CORT responses to nicotine were significant on the first but not the third day of SA in both strains. Chronic nicotine SA also enhanced the CORT response to mild foot shock in both strains. Therefore, strain differences may not be a major contributor to the mechanisms underlying (1) desensitization of the ACTH/CORT response to nicotine SA and (2) the enhanced ACTH/CORT response to mild foot shock during chronic nicotine SA. Although stressors were administered after a fixed dose and duration of exposure to nicotine SA, the minimum time and dose required to observe these differences is currently unknown.

These studies were designed to model human conditions, in which cigarettes are freely accessible to smokers who are exposed to stressors. Hence, nicotine was continuously available to rats both during and after the stress tests (except during immobilization stress). Since there was virtually no lever press activity during the brief stress sessions and the number of lever presses did not change significantly after foot shock, the enhancement in stress responsiveness induced by chronic nicotine SA is not likely to reflect any acute effects of nicotine.

In conclusion, the results reported herein support the concept that nicotine SA functions as a stressor. (1) The HPA axis was activated in rats by nicotine SA on the first day and this desensitized by the third day of $23 \mathrm{~h}$ access to the drug. (2) Chronic nicotine SA selectively enhanced the HPA responses to mild foot shock, but not to moderate foot shock nor to different dosages of LPS or immobilization. These effects of chronic nicotine SA on the HPA axis are congruent with those reported for other chronic stressors: namely, activation and desensitization of the HPA axis to a homotypic stressor, and cross-sensitization to a heterotypic stressor. Together, these data indicate that nicotine may increase the stress response to specific stressors in chronic cigarette smokers. This coheres with evidence that chronic smokers report higher perceived levels of stress.

\section{ACKNOWLEDGEMENTS}

This research was supported by DA-03977 (BMS) from NIDA. We thank Ms Kathy McAllen, Ms Weihua Cheng, and Dr Wenyuan Zhao for their technical contributions.

\section{DISCLOSURE/CONFLICT OF INTEREST}

The authors declare that there is no financial or other conflict of interest involving their biomedical research during the past 3 years.

\section{REFERENCES}

Andersson K, Siegel R, Fuxe K, Eneroth P (1983). Intravenous injections of nicotine induce very rapid and discrete reductions of hypothalamic catecholamine levels associated with increases of ACTH, vasopressin and prolactin secretion. Acta Physiol Scand 118: 35-40.

Armario A, Valles A, Dal-Zotto S, Marquez C, Belda X (2004). A single exposure to severe stressors causes long-term desensitisation of the physiological response to the homotypic stressor. Stress 7: 157-172.

Baer DS, McClearn GE, Wilson JR (1980). Effects of chronic administration of tobacco smoke to mice: behavioral and metabolic measures. Psychopharmacology (Berl) 67: 131-137.

Balfour DJ, Khullar AK, Longden A (1975). Effects of nicotine on plasma corticosterone and brain amines in stressed and unstressed rats. Pharmacol Biochem Behav 3: 179-184.

Benowitz NL, Jacob III P (1984). Daily intake of nicotine during cigarette smoking. Clin Pharmacol Ther 35: 499-504.

Benowitz NL, Jacob III P (1993). Nicotine and cotinine elimination pharmacokinetics in smokers and nonsmokers. Clin Pharmacol Ther 53: 316-323.

Bhatnagar S, Dallman M (1998). Neuroanatomical basis for facilitation of hypothalamic-pituitary-adrenal responses to a novel stressor after chronic stress. Neuroscience 84: 1025-1039.

Bhatnagar S, Huber R, Nowak N, Trotter P (2002). Lesions of the posterior paraventricular thalamus block habituation of hypothalamic-pituitary-adrenal responses to repeated restraint. J Neuroendocrinol 14: 403-410.

Brower VG, Fu Y, Matta SG, Sharp BM (2002). Rat strain differences in nicotine self-administration using an unlimited access paradigm. Brain Res 930: 12-20.

Caggiula AR, Donny EC, Epstein LH, Sved AF, Knopf S, Rose C et al (1998). The role of corticosteroids in nicotine's physiological and behavioral effects. Psychoneuroendocrinology 23: 143-159.

Cam GR, Bassett JR (1983a). The effect of acute nicotine administration on plasma levels of the thyroid hormones and corticosterone in the rat. Pharmacol Biochem Behav 19: 559-561.

Cam GR, Bassett JR (1983b). The plasma levels of ACTH following exposure to stress or nicotine. Arch Int Pharmacodyn Ther 264: 154-167.

Carey MP, Kalra DL, Carey KB, Halperin S, Richards CS (1993). Stress and unaided smoking cessation: a prospective investigation. J Consult Clin Psychol 61: 831-838.

Chassin L, Presson CC, Sherman SJ, Kim K (2002). Long-term psychological sequelae of smoking cessation and relapse. Health Psychol 21: 438-443.

Chuluyan HE, Saphier D, Rohn WM, Dunn AJ (1992). Noradrenergic innervation of the hypothalamus participates in adrenocortical responses to interleukin-1. Neuroendocrinology 56: 106-111.

Coan RW (1973). Personality variables associated with cigarette smoking. J Pers Soc Psychol 26: 86-104.

Cohen S, Lichtenstein E (1990). Perceived stress, quitting smoking, and smoking relapse. Health Psychol 9: 466-478.

Conte-Devolx B, Oliver C, Giraud P, Gillioz P, Castanas E, Lissitzky JC et al (1981). Effect of nicotine on in vivo secretion of melanocorticotropic hormones in the rat. Life Sci 28: 1067-1073.

Croghan IT, Bronars C, Patten CA, Schroeder DR, Nirelli LM, Thomas JL et al (2006). Is smoking related to body image satisfaction, stress, and self-esteem in young adults? Am J Health Behav 30: 322-333.

Dayas CV, Buller KM, Day TA (2004). Hypothalamic paraventricular nucleus neurons regulate medullary catecholamine cell responses to restraint stress. J Comp Neurol 478: 22-34.

Deak T, Bordner KA, McElderry NK, Barnum CJ, Blandino Jr P, Deak MM et al (2005). Stress-induced increases in hypothalamic 
IL-1: a systematic analysis of multiple stressor paradigms. Brain Res Bull 64: 541-556.

Donny EC, Caggiula AR, Rose C, Jacobs KS, Mielke MM, Sved AF (2000). Differential effects of response-contingent and responseindependent nicotine in rats. Eur J Pharmacol 402: 231-240.

Ericsson A, Kovacs KJ, Sawchenko PE (1994). A functional anatomical analysis of central pathways subserving the effects of interleukin-1 on stress-related neuroendocrine neurons. J Neurosci 14: 897-913.

Faraday MM, Blakeman KH, Grunberg NE (2005). Strain and sex alter effects of stress and nicotine on feeding, body weight, and HPA axis hormones. Pharmacol Biochem Behav 80: 577-589.

Faraday MM, O'Donoghue VA, Grunberg NE (2003). Effects of nicotine and stress on locomotion in Sprague-Dawley and LongEvans male and female rats. Pharmacol Biochem Behav 74: 325-333.

Finkelstein DM, Kubzansky LD, Goodman E (2006). Social status, stress, and adolescent smoking. J Adolesc Health 39: 678-685.

Fu Y, Matta SG, Brower VG, Sharp BM (2001). Norepinephrine secretion in the hypothalamic paraventricular nucleus of rats during unlimited access to self-administered nicotine: an in vivo microdialysis study. J Neurosci 21: 8979-8989.

Fu Y, Matta SG, Kane VB, Sharp BM (2003). Norepinephrine release in amygdala of rats during chronic nicotine selfadministration: an in vivo microdialysis study. Neuropharmacology 45: 514-523.

Gilbert DG, Meliska CJ, Williams CL, Jensen RA (1992). Subjective correlates of cigarette-smoking-induced elevations of peripheral beta-endorphin and cortisol. Psychopharmacology (Berl) 106: 275-281.

Goodwin GA, Bliven T, Kuhn C, Francis R, Spear LP (1997). Immediate early gene expression to examine neuronal activity following acute and chronic stressors in rat pups: examination of neurophysiological alterations underlying behavioral consequences of prenatal cocaine exposure. Physiol Behav 61: 895-902.

Kosten TA, Ambrosio E (2002). HPA axis function and drug addictive behaviors: insights from studies with Lewis and Fischer 344 inbred rats. Psychoneuroendocrinology 27: 35-69.

Koval JJ, Pederson LL, Chan SS (2004). Psychosocial variables in a cohort of students in grades 8 and 11: a comparison of current and never smokers. Prev Med 39: 1017-1025.

Lee AM, Miksys S, Palmour R, Tyndale RF (2006). CYP2B6 is expressed in African Green monkey brain and is induced by chronic nicotine treatment. Neuropharmacology 50: 441-450.

LeSage MG, Keyler DE, Shoeman D, Raphael D, Collins G, Pentel PR (2002). Continuous nicotine infusion reduces nicotine selfadministration in rats with 23 -h/day access to nicotine. Pharmacol Biochem Behav 72: 279-289.

Li HY, Ericsson A, Sawchenko PE (1996). Distinct mechanisms underlie activation of hypothalamic neurosecretory neurons and their medullary catecholaminergic afferents in categorically different stress paradigms. Proc Natl Acad Sci USA 93: 2359-2364.

Ma XM, Lightman SL, Aguilera G (1999). Vasopressin and corticotropin-releasing hormone gene responses to novel stress in rats adapted to repeated restraint. Endocrinology 140: 3623-3632.

Marks MJ, Burch JB, Collins AC (1983). Effects of chronic nicotine infusion on tolerance development and nicotinic receptors. J Pharmacol Exp Ther 226: 817-825.

Matta SG, Balfour DJ, Benowitz NL, Boyd RT, Buccafusco JJ, Caggiula AR et al (2007). Guidelines on nicotine dose selection for in vivo research. Psychopharmacology (Berl) 190: 269-319.

Matta SG, Beyer HS, McAllen KM, Sharp BM (1987). Nicotine elevates rat plasma ACTH by a central mechanism. J Pharmacol Exp Ther 243: 217-226.

Matta SG, Fu Y, Valentine JD, Sharp BM (1998). Response of the hypothalamo-pituitary-adrenal axis to nicotine. Psychoneuroendocrinology 23: 103-113.
Matta SG, McCoy JG, Foster CA, Sharp BM (1995). Nicotinic agonists administered into the fourth ventricle stimulate norepinephrine secretion in the hypothalamic paraventricular nucleus: an in vivo microdialysis study. Neuroendocrinology 61: 383-392.

Matta SG, Singh J, Sharp BM (1990). Catecholamines mediate nicotine-induced adrenocorticotropin secretion via alpha-adrenergic receptors. Endocrinology 127: 1646-1655.

McArthur C, Waldron E, Dickinson J (1958). The psychology of smoking. J Abnorm Psychol 56: 267-275.

Mendelson JH, Sholar MB, Goletiani N, Siegel AJ, Mello NK (2005). Effects of low- and high-nicotine cigarette smoking on mood states and the HPA axis in men. Neuropsychopharmacology 30: 1751-1763.

Pagliusi SR, Tessari M, DeVevey S, Chiamulera C, Pich EM (1996). The reinforcing properties of nicotine are associated with a specific patterning of $\mathrm{c}$-fos expression in the rat brain. Eur $J$ Neurosci 8: 2247-2256.

Parker SL, Fu Y, McAllen K, Luo J, McIntosh JM, Lindstrom JM et al (2004). Up-regulation of brain nicotinic acetylcholine receptors in the rat during long-term self-administration of nicotine: disproportionate increase of the alpha6 subunit. $\mathrm{Mol}$ Pharmacol 65: 611-622.

Parrott AC (1995). Stress modulation over the day in cigarette smokers. Addiction 90: 233-244.

Passerin AM, Cano G, Rabin BS, Delano BA, Napier JL, Sved AF (2000). Role of locus coeruleus in foot shock-evoked Fos expression in rat brain. Neuroscience 101: 1071-1082.

Radley JJ, Arias CM, Sawchenko PE (2006). Regional differentiation of the medial prefrontal cortex in regulating adaptive responses to acute emotional stress. J Neurosci 26: 12967-12976.

Ray MA, Graham AJ, Lee M, Perry RH, Court JA, Perry EK (2005). Neuronal nicotinic acetylcholine receptor subunits in autism: an immunohistochemical investigation in the thalamus. Neurobiol Dis 19: 366-377.

Sawchenko PE, Li HY, Ericsson A (2000). Circuits and mechanisms governing hypothalamic responses to stress: a tale of two paradigms. Prog Brain Res 122: 61-78.

Schepis TS, Rao U (2005). Epidemiology and etiology of adolescent smoking. Curr Opin Pediatr 17: 607-612.

Sharp BM, Beyer HS (1986). Rapid desensitization of the acute stimulatory effects of nicotine on rat plasma adrenocorticotropin and prolactin. J Pharmacol Exp Ther 238: 486-491.

Stier Jr CT, Serova LI, Singh G, Sabban EL (2004). Stress triggered rise in plasma aldosterone is lessened by chronic nicotine infusion. Eur J Pharmacol 495: 167-170.

Stone EA, Lehmann ML, Lin Y, Quartermain D (2006). Depressive behavior in mice due to immune stimulation is accompanied by reduced neural activity in brain regions involved in positively motivated behavior. Biol Psychiatry 60: 803-811.

Takemura T, Makino S, Takao T, Asaba K, Suemaru S, Hashimoto K (1997). Hypothalamic-pituitary-adrenocortical responses to single $v s$ repeated endotoxin lipopolysaccharide administration in the rat. Brain Res 767: 181-191.

Valentine JD, Hokanson JS, Matta SG, Sharp BM (1997). Selfadministration in rats allowed unlimited access to nicotine. Psychopharmacology (Berl) 133: 300-304.

Wan W, Wetmore L, Sorensen CM, Greenberg AH, Nance DM (1994). Neural and biochemical mediators of endotoxin and stress-induced c-fos expression in the rat brain. Brain Res Bull 34: 7-14.

Zhao R, Chen H, Sharp BM (2007). Nicotine-Induced Norepinephrine Release in Hypothalamic Paraventricular Nucleus and Amygdala Is Mediated by N-Methyl-D-aspartate Receptors and Nitric Oxide in the Nucleus Tractus Solitarius. J Pharmacol Exp Ther 320: 837-844. 